\title{
RECONSTRUCTION OF LESS REGULAR CONDUCTIVITIES IN THE PLANE
}

\author{
KIM KNUDSEN AND ALEXANDRU TAMASAN
}

\begin{abstract}
We study the inverse conductivity problem of how to reconstruct an isotropic electrical conductivity distribution $\gamma$ in an object from static electrical measurements on the boundary of the object. We give an exact reconstruction algorithm for the conductivity $\gamma \in C^{1+\epsilon}(\bar{\Omega})$ in the plane domain $\Omega$ from the associated Dirichlet to Neumann map on $\partial \Omega$. Hence we improve earlier reconstruction results. The method used relies on a well-known reduction to a first order system, for which the $\bar{\partial}$-method of inverse scattering theory can be applied.
\end{abstract}

\section{INTRODUCTION}

Let $\Omega \subset \mathbb{R}^{n}$ be a bounded and smooth domain and let $\gamma \in L^{\infty}(\Omega)$ with $0<c \leq$ $\gamma(x)$, a.e. $x \in \Omega$ for some constant $c$. Consider the conductivity problem

$$
\begin{array}{r}
\nabla \cdot \gamma \nabla u=0, \text { in } \Omega, \\
u=f, \text { on } \partial \Omega,
\end{array}
$$

for $f \in H^{1 / 2}(\partial \Omega)$. This problem has a unique solution $u \in H^{1}(\Omega)$ and hence we can define the Dirichlet to Neumann map

$$
\begin{aligned}
\Lambda_{\gamma}: H^{1 / 2}(\partial \Omega) & \rightarrow H^{-1 / 2}(\partial \Omega), \\
f & \left.\mapsto \gamma \frac{\partial u}{\partial \nu}\right|_{\partial \Omega},
\end{aligned}
$$

where $\nu$ is the outer unit normal defined in a neighborhood of $\partial \Omega$. This definition should be understood in the sense, that if $f, g \in H^{1 / 2}(\partial \Omega)$, then

$$
\left\langle\Lambda_{\gamma} f, g\right\rangle=\int_{\Omega} \gamma \nabla u \cdot \nabla v d x,
$$

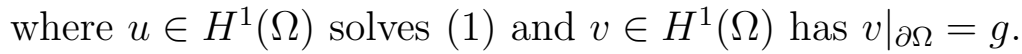

In this paper we study the problem of reconstructing $\gamma$ from knowledge of $\Lambda_{\gamma}$. This is the reconstruction issue of the inverse conductivity problem.

The main motivation for studying this problem comes from a method for medical imaging called Electrical Impedance Tomography (EIT) (see [CIN99] for a thorough review of current methods in EIT). In EIT one has a conductive body $\Omega$ with unknown conductivity $\gamma$, and from static electric measurements on the boundary, i.e. by applying a voltage potential on $\partial \Omega$ and measuring the current flux through $\partial \Omega$, one would like to monitor the interior conductivity. Since tissue, bones, lungs etc.

Research at MSRI is supported in part by Aalborg University, Denmark, and in part by NSF grant DMS-0070488. 
have different conductive properties, an image of the conductivity is an image of the internal structures.

The mathematical formulation of the inverse conductivity problem was introduced by Calderón in [Cal80]. Calderón gave an approximate reconstruction method for $\gamma$ close to constant. Since Calderón's fundamental paper a lot of work has been done on this problem.

The first global result was in $\left[K \nabla 84\right.$, where it was proved, that if $\gamma \in C^{\infty}(\bar{\Omega})$ then $\Lambda_{\gamma}$ determines $\left.\gamma\right|_{\partial \Omega}$ and all partial derivatives on $\partial \Omega$. From here it follows by analytic continuation, that if $\gamma$ is real-analytic in $\Omega$ then it is determined by $\Lambda_{\gamma}$. Later in KV85 the result was extended to include piecewise real-analytic conductivities.

In three dimensions a major breakthrough in the theory of inverse boundary value problems was the paper [SU87]. There it was proved that $\Lambda_{\gamma}$ determines $\gamma \in C^{\infty}(\bar{\Omega})$. Their method of proof relies on the existence of special solutions to the conductivity equation, the so-called complex geometrical optics solutions or exponentially growing solutions, which behave like $e^{x \cdot \zeta}$ for large $|x|$ and for $\zeta \in \mathbb{C}^{n}$ with $\zeta \cdot \zeta=0$. The assumption on $\gamma$ has since been relaxed by a number of authors ([Ale88], Nac88], [NSU88], Bro96]). The best uniqueness result so far is for $\gamma \in C^{3 / 2}(\bar{\Omega})$ obtained in [PPD]. Using exponentially growing solutions Nachman $\left([\mathrm{Nac} 88]\right.$ ) gave a reconstruction algorithm for conductivities $\gamma \in C^{1,1}(\bar{\Omega})$. Furthermore, Alessandrini ( Ale88 ) proved a conditional stability result.

In two dimensions, uniqueness for small $\gamma \in W^{3, \infty}(\Omega)$ was proved in [SU86], but the general question remained open until 1996, when Nachman Nac96 gave a uniqueness proof and a reconstruction algorithm for $\gamma \in W^{2, p}(\Omega), p>1$. Nachman's method relies on the reduction of the conductivity equation to a Schrödinger equation, which essentially requires two derivatives of the conductivity. For this equation he proved existence of exponentially growing solution, which can be reconstructed from $\Lambda_{\gamma}$ by the so-called $\bar{\partial}$-method in inverse scattering theory originally developed by Beals and Coifman (see [BC89] and the reference there). Then using the exponentially growing solutions Nachman was able to reconstruct $\gamma$. The algorithm has been tried out numerically (see [SMI00]). In 1997 Brown and Uhlmann ([BU97]) improved the uniqueness result for $\gamma \in W^{1, p}(\Omega), p>2$. Their method relies on a reduction to a first order system for which only one derivative is required. For this system the $\bar{\partial}$-method is again applicable, see Section 2 for further details. For both works conditional stability has been proved, see [Liu97 and BBR01.

For a thorough review on the history and developments of the inverse conductivity problem we refer to Uhl99.

In this paper we will show how the uniqueness proof in $B 097$ for the twodimensional inverse conductivity problem can be turned into a reconstruction method. The reconstruction algorithm parallels Nachman's algorithm, but since the reduction used here requires less regularity, we can improve the reconstruction result in Nac96]. The main result is the following:

Theorem 1.1. Let $\Omega \subset \mathbb{R}^{2}$ be a bounded and smooth domain. Let $\epsilon>0$ and let $\gamma \in$ $C^{1+\epsilon}(\bar{\Omega})$ be such that $0<c \leq \gamma(x)$ for some constant $c$. Then $\gamma$ can be reconstructed from $\Lambda_{\gamma}$. 
The proof is given in the following sections as follows: In Section 2 we introduce notation, outline the main idea of the uniqueness proof and give the reconstruction algorithm. The steps of the algorithm are then justified in the following sections.

\section{Outline of the ReCOnstruction}

In this section we introduce notations and review the reduction of (1) to a first order system. We then formulate an inverse problem for this system and finally outline the reconstruction method.

Generally we work under the hypothesis stated in Theorem 1.1. Furthermore, we assume that $\gamma=1$ near $\partial \Omega$. It was noticed by Nachman in Nac96, that this assumption is not a restriction. When $\gamma \in C^{1+\epsilon}(\bar{\Omega})$ it is well-known ( Ale88, , SU88, , Nac88) that $\gamma$ and its normal derivative on the boundary are determined by $\Lambda_{\gamma}$. Hence $\gamma$ can be extended outside $\Omega$, such that it is identically 1 outside a compact set and $\gamma \in C^{1+\epsilon}\left(\mathbb{R}^{2}\right)$. Moreover, the Dirichlet to Neumann map on the boundary of a larger domain containing the original domain can be reconstructed from the original Dirichlet to Neumann map and from the known extension. We show in the appendix how to do such a construction for $\gamma \in L^{\infty}(\Omega)$.

It is well-known from the theory of elliptic equations, that if $f \in C^{2+\epsilon}(\partial \Omega)$ and $\gamma \in C^{1+\epsilon}(\bar{\Omega})$, then (1) has a unique solution $u \in C^{2+\epsilon}(\bar{\Omega})$. Then the vector valued function $(v, w)^{T}$ defined by

$$
\left(\begin{array}{c}
v \\
w
\end{array}\right)=\gamma^{1 / 2}\left(\begin{array}{c}
\partial u \\
\bar{\partial} u
\end{array}\right) \in C^{1+\epsilon}(\bar{\Omega}) \times C^{1+\epsilon}(\bar{\Omega})
$$

satisfies the equation

$$
(D-Q)\left(\begin{array}{c}
v \\
w
\end{array}\right)=0 .
$$

Here the matrix operator $D$ and the matrix potential $Q$ are defined by

$$
D=\left(\begin{array}{ll}
\bar{\partial} & 0 \\
0 & \partial
\end{array}\right), \quad Q=\left(\begin{array}{cc}
0 & q \\
\bar{q} & 0
\end{array}\right),
$$

where $\partial=\left(\partial_{x}-i \partial_{y}\right) / 2$ and $\bar{\partial}=\left(\partial_{x}+i \partial_{y}\right) / 2$ and

$$
q=-\gamma^{-1 / 2} \partial \gamma^{1 / 2}
$$

We shall see below in the proof of Theorem 3.2 that there is a one-to-one correspondence between $u$ and $(v, w)^{T}$, i.e. given a solution $(v, w)$ to (3), where $Q$ is of the form (14) with $q$ given by (5), there exists a solution $u$ to (11) such that (2) holds.

With $Q \in C^{\epsilon}\left(\mathbb{R}^{2}\right)$, the natural Cauchy data for the system (3) is

$$
\mathcal{C}_{Q}=\left\{\left(\left.v\right|_{\partial \Omega},\left.w\right|_{\partial \Omega}\right):(v, w) \in C^{1+\epsilon}(\bar{\Omega}) \times C^{1+\epsilon}(\bar{\Omega}),(D-Q)(v, w)^{T}=0\right\} .
$$

We can now pose the following inverse problem for the system (3): Let $q \in C^{\epsilon}(\bar{\Omega})$ and $Q$ defined by (四). Is $Q$ uniquely determined from $\mathcal{C}_{Q}$ and, in case it is, how can it be reconstructed?

In this paper we show how to reconstruct $Q$ coming from a conductivity equation. For this particular case we give an explicit characterization of the Cauchy data $\mathcal{C}_{Q}$ in 
terms of the Dirichlet to Neumann map $\Lambda_{\gamma}$ and this characterization enables us to solve the inverse problem.

Brown and Uhlmann ([Bण97]) were the first to use (3) in the study of the inverse conductivity problem and their uniqueness proof was based on the scattering theory for this system developed by Beals and Coifman ([BC88]). For the direct scattering, one looks for a family of functions, which solve the equation (3) in the whole plane (with $Q$ extended by zero outside $\Omega$ ) and have a certain exponential behaviour at infinity. More precisely, for each $k \in \mathbb{C}$, one seek solutions $\Psi(\cdot, k)$ of the form

$$
\Psi(z, k)=m(z, k)\left(\begin{array}{cc}
e^{i z k} & 0 \\
0 & e^{-i \bar{z} k}
\end{array}\right),
$$

where $m$ approaches the identity matrix $I$ as $|z| \rightarrow \infty$ and $z=x_{1}+i x_{2} \in \mathbb{C}$. These solutions are called exponentially growing solutions or complex geometrical optics.

A simple calculations shows that $m$ should solve the equation

$$
\left(D_{k}-Q\right) m=0
$$

where $D_{k}$ is the matrix operator

$$
D_{k}=\left(\begin{array}{cc}
(\bar{\partial}-i \bar{k}) & 0 \\
0 & (\partial+i k)
\end{array}\right) .
$$

The analysis of the direct scattering problem along these lines is due to Beals and Coifman ( $\mathrm{BC}$ 85], [BC88]). A detailed analysis for the case $Q \in L^{1}\left(\mathbb{R}^{2}\right) \cap L^{\infty}\left(\mathbb{R}^{2}\right)$ can be found in [Sun94a, Sun94b], Sun94d, and the analysis for the case of compactly supported $Q \in L^{p}\left(\mathbb{R}^{2}\right), p>2$, can be found in $[\mathrm{BU}$-96]. Given our assumptions on $Q$ we have the following theorem, see BU96] and BBR01.

Theorem 2.1. Let $Q$ be a hermitian, compactly supported matrix with $Q \in C^{\epsilon}\left(\mathbb{R}^{2}\right)$. Then for any $2<r<\infty$ and $k \in \mathbb{C}$ there is a unique solution $m(z, k)$ to (7) with $(m(\cdot, k)-I) \in L^{r}\left(\mathbb{R}^{2}\right) \cap C^{\epsilon}\left(\mathbb{R}^{2}\right)$.

Furthermore, for fixed $z \in \mathbb{R}^{2}$, the map $k \mapsto m(z, k)$ is differentiable, and it satisfies the equation

$$
\frac{\partial}{\partial \bar{k}} m(z, k)=m(z, \bar{k}) \Lambda_{k}(z) S(k)
$$

where

$$
S(k)=\frac{i}{\pi} \int_{\mathbb{C}}\left(\begin{array}{cc}
0 & q(z) m_{22}(z, k) \\
-\bar{q}(z) m_{11}(z, k) & 0
\end{array}\right) d \mu(z)
$$

and

$$
\Lambda_{k}(z)=\left(\begin{array}{cc}
e(z, \bar{k}) & 0 \\
0 & e(z,-k)
\end{array}\right)
$$

for $e(z, k)=\exp (i z k+i \overline{z k})$.

In the formula (9) $m_{11}$ and $m_{22}$ are the diagonal components of $m$, and $d \mu(z)$ is the usual area measure. Note, that since $Q \in C^{\epsilon}\left(\mathbb{R}^{2}\right)$, elliptic regularity theory gives, that $m \in C_{l o c}^{1+\epsilon}\left(\mathbb{R}^{2}\right)$. This fact will be used later. 
The matrix $S(k)$ is called the scattering matrix. Using the definition of $\Psi$ and the fact that $Q$ is supported in $\Omega$, an integration by parts in (9) shows that

$$
S(k)=\frac{i}{2 \pi} \int_{\partial \Omega}\left(\begin{array}{cc}
0 & e^{-i z \bar{k}} \nu(z) \psi_{12}(z, k) \\
-e^{i z k} \bar{\nu}(z) \psi_{21}(z, k) & 0
\end{array}\right) d \sigma(z),
$$

where $\psi_{12}$ and $\psi_{21}$ are the off diagonal entries of $\Psi$. In the above formula, $d \sigma(z)$ is the Lebesgue measure on the boundary and $\nu$ is the complex normal at the boundary, i.e., if $\left(\nu_{1}(z), \nu_{2}(z)\right)$ denotes the outer unit normal at $z \in \partial \Omega$, then $\nu(z)=\nu_{1}(z)+i \nu_{2}(z)$ and $\bar{\nu}=\nu_{1}(z)-i \nu_{2}(z)$. Therefore, in order to determine the scattering matrix $S(k)$, it suffices to find the traces on $\partial \Omega$ of the exponentially growing solutions.

Once $S(k)$ is known, we can solve the $\bar{\partial}$-equation (8) to find $m(z, k)$ for each fixed $z \in \mathbb{C}$ and reconstruct $Q$ by the formula

$$
Q(z)=\lim _{k_{0} \rightarrow \infty} \mu\left(B_{r}(0)\right)^{-1} \int_{\left\{k:\left|k-k_{0}\right|<r\right\}} D_{k} m(z, k) d \mu(k)
$$

from [BU97]. This would solve the inverse problem for (3). Furthermore, $\gamma$ can be found by solving (5).

Since we are mainly interested in reconstructing the conductivity, and this approach then requires taking the parameter to infinity and solving a boundary value problem, we shall take a different route. As observed in [BBR01] we can reconstruct $\gamma$ directly from $m(z, 0)$. In fact we will see, that

$$
\gamma^{1 / 2}(z)=\operatorname{Re}\left(\tilde{m}_{11}(z, 0)+\tilde{m}_{21}(z, 0)\right)
$$

where now $\tilde{m}$ is the unique solution given by Theorem 2.1 to $D_{k} \tilde{m}=-Q^{T} m$. It was noticed by Beals and Coifman that the scattering matrix $\tilde{S}$ associated the potential $-Q^{T}$ relates to the scattering matrix $S$ for $Q$ by the formula

$$
\tilde{S}(k)=S(-\bar{k})^{T} \text {. }
$$

Therefore $\tilde{m}$ can be found from $S$ by solving (8) with $S$ replaced by $S(-\bar{k})^{T}$.

We are now ready to outline the reconstruction method:

1. Determine $\Psi$ on $\partial \Omega$ (see Theorem 4.3 below)

2. Define the scattering matrix $S$ by $(10)$ and $\tilde{S}$ by (12)

3. Solve the $\bar{\partial}$-equation (8) with $\tilde{S}$ for $\tilde{m}(z, k), z \in \Omega$

4. Recover $\gamma$ on $\Omega$ from (11).

The implementation of this method is subject to further study.

\section{A BOUNDARY RELATION}

In this section we give an explicit characterization of the Cauchy data for the first order system (3), in case the potential $Q$ comes from a conductivity.

Assume $u \in C^{2+\epsilon}(\bar{\Omega})$ solves (11) for some $f \in C^{2+\epsilon}(\partial \Omega)$. Then $(v, w)=\gamma^{1 / 2}(\partial u, \bar{\partial} u)$ solves (3) and one easily finds the relation

$$
\left.\left(\begin{array}{c}
v \\
w
\end{array}\right)\right|_{\partial \Omega}=\frac{1}{2}\left(\begin{array}{cc}
\bar{\nu} & -i \bar{\nu} \\
\nu & i \nu
\end{array}\right)\left(\begin{array}{c}
\Lambda_{\gamma}(f) \\
\partial_{s}(f)
\end{array}\right) \text {, on } \partial \Omega
$$


where $\partial_{s}$ denotes the tangential derivative on $\partial \Omega$. Inverting (13) gives

$$
\left(\begin{array}{c}
\Lambda_{\gamma}(f) \\
\partial_{s}(f)
\end{array}\right)=\left.\left(\begin{array}{cc}
\nu & \bar{\nu} \\
i \nu & -i \bar{\nu}
\end{array}\right)\left(\begin{array}{c}
v \\
w
\end{array}\right)\right|_{\partial \Omega}
$$

Let $\partial_{s}^{-1}: C^{1+\epsilon}(\partial \Omega) \rightarrow C^{2+\epsilon}(\partial \Omega)$ denote the normalized inverse of $\partial_{s}$ on the boundary defined by

$$
\partial_{s}^{-1} f(s)=\int_{0}^{s} f d s+c(f)
$$

where the measure $d s$ is the arc length on $\partial \Omega$ and the constant $c(f)$ is chosen so that $\int_{\partial \Omega} \partial_{s}^{-1} f d s=0$. Then from (14) we arrive at the following boundary relation for $\left.(v, w)\right|_{\partial \Omega}$

$$
i \Lambda_{\gamma} \partial_{s}^{-1}\left(\left.\nu v\right|_{\partial \Omega}-\left.\bar{\nu} w\right|_{\partial \Omega}\right)=\left(\left.\nu v\right|_{\partial \Omega}+\left.\bar{\nu} w\right|_{\partial \Omega}\right) .
$$

This relation motivates the following definition

Definition 3.1. Let $\left(h_{1}, h_{2}\right)$ be a pair of functions in $C^{1+\epsilon}(\partial \Omega) \times C^{1+\epsilon}(\partial \Omega)$. We say that $\left(h_{1}, h_{2}\right)$ satisfies the boundary relation if

$$
i \Lambda_{\gamma} \partial_{s}^{-1}\left(\nu h_{1}-\bar{\nu} h_{2}\right)=\left(\nu h_{1}+\bar{\nu} h_{2}\right) .
$$

We just saw that solutions to (3), defined through (2), satisfy the boundary relation, but as the following theorem shows, (15) is in fact a complete characterization of $\mathcal{C}_{Q}$ :

Theorem 3.2. If $Q$ is given by (5) then

$$
\mathcal{C}_{Q}=\left\{\left(h_{1}, h_{2}\right) \in C^{1+\epsilon}(\partial \Omega) \times C^{1+\epsilon}(\partial \Omega): i \Lambda_{\gamma} \partial_{s}^{-1}\left(\nu h_{1}-\bar{\nu} h_{2}\right)=\nu h_{1}+\bar{\nu} h_{2}\right\} .
$$

Proof. First we show that any pair $\left(h_{1}, h_{2}\right) \in C^{1+\epsilon}(\partial \Omega) \times C^{1+\epsilon}(\partial \Omega)$ which satisfies the boundary relation is in $\mathcal{C}_{Q}$. Let $u \in C^{2+\epsilon}(\bar{\Omega})$ be the unique solution of the Dirichlet problem

$$
\begin{cases}\nabla \cdot \gamma \nabla u=0, & \text { in } \Omega, \\ u=i \partial_{s}^{-1}\left(\nu h_{1}-\bar{\nu} h_{2}\right), & \text { in } \partial \Omega .\end{cases}
$$

Define a solution $(v, w)^{T}$ to (3) by the relation (2) with $u$ from above. That $\left.(v, w)\right|_{\partial \Omega}=$ $\left(h_{1}, h_{2}\right)$ follows from (13) by the use of (15)).

Conversely, we start with a solution $(v, w) \in C^{1+\epsilon}(\bar{\Omega}) \times C^{1+\epsilon}(\bar{\Omega})$ to (3) and check the boundary relation for $\left.(v, w)\right|_{\partial \Omega}$. Since $v$ and $w$ solve the linear system (3) and $Q$ is of the form (5), we have the compatibility relation

$$
\bar{\partial}\left(\gamma^{-1 / 2} v\right)=\partial\left(\gamma^{-1 / 2} w\right)
$$

The Poincaré lemma ensures now the existence of such a $u$ that

$$
\gamma^{-1 / 2}\left(\begin{array}{c}
v \\
w
\end{array}\right)=\left(\begin{array}{l}
\partial u \\
\bar{\partial} u
\end{array}\right)
$$

It is easy to check that $u$ is a solution to the conductivity equation in the form $2 \partial u \bar{\partial} \gamma+2 \bar{\partial} u \partial \gamma+4 \gamma \partial \bar{\partial} u=0$. Now relation (13) with $f=\left.u\right|_{\partial \Omega}$ shows that $\left(h_{1}, h_{2}\right)$ solves the boundary relation. 


\section{From $\Lambda_{\gamma}$ TO $S(k)$}

In this section we show how to reconstruct the trace on $\partial \Omega$ of the exponentially growing solutions $\Psi(z, k)$ defined by (6). Notice that the first row has entries which are analytic outside $\Omega$, while the second row has entries which are anti-analytic outside $\Omega$. Moreover they have a prescribed behavior at infinity and their traces on $\partial \Omega$ satisfy the boundary relation (15). We will prove, that these relations are sufficient to reconstruct the trace of $\Psi$ uniquely.

Due to the symmetries

$$
m_{11}(z, k)=\overline{m_{22}(z, \bar{k})}, \quad m_{21}(z, k)=\overline{m_{12}(z, \bar{k})},
$$

which follows from the differential equations, the asymptotic for the columns of $m$ and the uniqueness in Theorem 2.1, it suffices to reconstruct the first column $\left(\Psi_{11}, \Psi_{21}\right)^{T}$ of $\Psi(z, k)$.

For every $(\zeta, z) \in \mathbb{C}$ with $\zeta \neq z$ we introduce

$$
g_{k}(\zeta, z)=\frac{1}{\pi} \frac{e^{-i k(\zeta-z)}}{\zeta-z}
$$

a Green's kernel for $\bar{\partial}$ which also takes into account exponential grow at infinity. Using $g_{k}(\cdot, z)$ with $z \in \partial \Omega$, we define the single layer potentials $\mathcal{S}_{k}$ and $\overline{\mathcal{S}}_{k}$ as boundary integral operators by

$$
\mathcal{S}_{k} f(z)=\int_{\partial \Omega} f(\zeta) g_{k}(\zeta, z) d \zeta, \overline{\mathcal{S}}_{k} f(z)=\int_{\partial \Omega} f(\zeta) \bar{g}_{k}(\zeta, z) d \zeta
$$

These integrals are understood in the sense of principal value. It is a classical result in singular integral theory that these operators are well defined, see Mus53.

Proposition 4.1. If $0<\epsilon<1$ then $\mathcal{S}_{k}, \overline{\mathcal{S}}_{k}: C^{1+\epsilon}(\partial \Omega) \rightarrow C^{1+\epsilon}(\partial \Omega)$ are well defined bounded operators.

Let $\mathcal{K}$ denote the matrix operator

$$
\mathcal{K}=\frac{1}{2}\left(\begin{array}{cc}
I-i \mathcal{S}_{k} & 0 \\
0 & I-\overline{\mathcal{S}}_{k}
\end{array}\right)
$$

The following lemma gives a necessary condition for a pair of functions defined in $\Omega^{c}=\mathbb{C} \backslash \bar{\Omega}$ to be analytic and anti-analytic respectively and have a special exponential decay at infinity:

Proposition 4.2. Let $v$ be analytic and $w$ be anti-analytic in $\Omega^{c}$ and continuous to $\partial \Omega$, such that $v e^{-i z k}-1 \in L^{r}\left(\Omega^{c}\right)$ and $w e^{-i z k} \in L^{r}\left(\Omega^{c}\right)$ for some $r \geq 1$. Then the trace $\left(h_{1}, h_{2}\right)=\left.(v, w)\right|_{\partial \Omega}$ solves

$$
\mathcal{K}\left(\begin{array}{l}
h_{1} \\
h_{2}
\end{array}\right)=\left(\begin{array}{c}
e^{i z k} \\
0
\end{array}\right) .
$$

Proof. We will prove the relation for $v$; for $w$ the result follows by similar arguments. Let $z \in \Omega^{c}$ be a fixed point inside some large ball $B_{R}$ of radius $R$. With the $\bar{\partial}$ 
operator acting with respect to $\zeta$ and $R>0$ such that $\bar{\Omega} \subset B(0, R)$, Pompeiu's formula $([$ Vek62] $)$ states, that

$$
\begin{aligned}
v(z) e^{-i k z}-1= & \frac{1}{2 \pi i} \int_{\partial B_{R}} \frac{v(\zeta) e^{-i k \zeta}-1}{\zeta-z} d \zeta-\frac{1}{2 \pi i} \int_{\partial \Omega} \frac{v(\zeta) e^{-i k \zeta}-1}{\zeta-z} d \zeta \\
& -\int_{B_{R} \backslash \bar{\Omega}} \frac{\bar{\partial}\left(v(\zeta) e^{-i k \zeta}-1\right)}{\zeta-z} d \zeta \\
= & \frac{1}{2 \pi i} \int_{\partial B_{R}} \frac{v(\zeta) e^{-i k \zeta}-1}{\zeta-z} d \zeta-\frac{1}{2 \pi i} \int_{\partial \Omega} \frac{v(\zeta) e^{-i k \zeta}-1}{\zeta-z} d \zeta .
\end{aligned}
$$

Since $v e^{-i z k}-1 \in L^{r}\left(\mathbb{R}^{2}\right)$ the integral on $\partial B_{R}$ converges to zero at least on a sequence of increasing radii, and since $z \in \Omega^{c}$, Cauchy's formula gives

$$
\frac{1}{2 \pi i} \int_{\partial \Omega} \frac{1}{\zeta-z} d \zeta=0
$$

Therefore

$$
v(z)+\frac{1}{2 i} \int_{\partial \Omega} v(\zeta) g_{k}(\zeta, z) d \zeta=e^{i z k}, z \in \Omega^{c} .
$$

Now let $z \in \Omega^{c}$ approach some point on the boundary and apply Plemelj's Formula Mus53 to get the equation for $v$.

We know that the exponentially growing solutions $\left.\left(\psi_{11}(\cdot, k), \psi_{21}(\cdot, k)\right)\right|_{\partial \Omega}$ must satisfy (19) and the boundary relation (15). The following result shows that these conditions are sufficient to characterize the trace of the exponentially growing solutions.

Theorem 4.3. The only pair $\left(h_{1}, h_{2}\right)^{T} \in C^{1+\epsilon}(\partial \Omega) \times C^{1+\epsilon}(\partial \Omega)$ which satisfies

$$
\begin{aligned}
\mathcal{K}\left(\begin{array}{l}
h_{1} \\
h_{2}
\end{array}\right) & =\left(\begin{array}{c}
e^{i z k} \\
0
\end{array}\right) \\
\left(I-i \Lambda_{\gamma} \partial_{s}^{-1}\right)\left(\nu h_{1}\right)(z) & =\left(I+i \Lambda_{\gamma} \partial_{s}^{-1}\right)\left(\bar{\nu} h_{2}\right)(z),
\end{aligned}
$$

is $\left.\left(\psi_{11}(\cdot, k), \psi_{21}(\cdot, k)\right)\right|_{\partial \Omega}$.

Proof. Let $h=\left(h_{1}, h_{2}\right) \in C^{1+\epsilon}(\partial \Omega) \times C^{1+\epsilon}(\partial \Omega)$ be any solution to (21). Guided by (20) and the related formula for $w$ we extend $h$ outside $\Omega$ to $(v(z), w(z))$ by

$$
v(z)=-\frac{1}{2 i} \int_{\partial \Omega} h_{1}(\zeta) g_{k}(\zeta, z) d \zeta+e^{i z k}, w(z)=-\frac{1}{2 i} \int_{\partial \Omega} h_{2}(\zeta) \bar{g}_{k}(\zeta, z) d \zeta .
$$

Inside $\Omega$ we extend $h$ as a solution to (3), which can be done by Theorem 4.3, since $h$ satisfies the boundary relation.

We will prove, that $(v, w)$ is a solution to (3) and that $v e^{-i z k}-1, w \in L^{r}\left(\mathbb{R}^{2}\right), r>2$. Then the result follows from Theorem 2.1.

Outside $\Omega, v$ is analytic and $w$ is anti-analytic. Moreover, since $\gamma$ is identically 1 near the boundary, we also have that $v$ is analytic and $w$ is anti-analytic in an inside neighborhood of the boundary. We need to check the analyticity of $v$ and antianalyticity of $w$ as we go across the boundary. For this it suffices to have continuity 
across the boundary and then invoke Morera's theorem. We shall show the continuity of $v$, for $w$ similar reasoning works. Let $z$ approach some point $z_{0} \in \partial \Omega$ from outside. Then using Plemelj's formula we get

$$
\lim _{z \rightarrow z_{0}^{+}} v(z)=-\left(-\frac{h_{1}\left(z_{0}\right)}{2}+\frac{1}{2 i} S_{k} h_{1}\left(z_{0}\right)\right)+e^{i z_{0} k}
$$

Now use the first equation of (21) to conclude $\lim _{z \rightarrow z_{0}} v(z)=h_{1}\left(z_{0}\right)$. The continuity of $v$ from inside comes from the regularity theory for elliptic equations.

We are left to show that $v e^{-i z k}-1 \in L^{r}\left(\mathbb{R}^{2}\right)$, but since

$$
v(z) e^{-i z k}-1=-\frac{1}{2 \pi i} \int_{\partial \Omega} \frac{h_{1}(\zeta) e^{-i k \zeta}}{\zeta-z} d \zeta=O\left(\frac{1}{|z|}\right), \text { as }|z| \rightarrow \infty,
$$

it is in $L^{r}\left(\mathbb{R}^{2}\right)$ for $r>2$. This proves the theorem.

As an corollary we easily get

Corollary 4.4. $\operatorname{ker} \mathcal{K} \cap \mathcal{C}_{Q}=\{0\}$.

Proof. Let $\left(h_{1}, h_{2}\right)^{T} \in \operatorname{ker} \mathcal{K} \cap \mathcal{C}_{Q}$ and $(v, w)^{T}$ be the trace of the exponentially growing solutions. Then $\left(h_{1}+v, h_{2}+w\right)^{T}$ is also satisfies the boundary relation and satisfies (21). Now use the uniqueness of Theorem 4.3 to conclude that $\left(h_{1}, h_{2}\right)^{T}=0$.

To obtain the exponentially growing solutions on $\partial \Omega$ one has to solve the three equations (21). Since $\left(e^{i k z}, 0\right)^{T}$ solves

$$
\mathcal{K}\left(\begin{array}{c}
e^{i z k} \\
0
\end{array}\right)=\left(\begin{array}{c}
e^{i z k} \\
0
\end{array}\right)
$$

the pair of traces of the exponentially growing solutions can be constructed as the projection of $\left(e^{i k z}, 0\right)^{T}$ on $\mathcal{C}_{Q}$ along ker $\mathcal{K}$.

\section{FROM $S(k)$ TO $\gamma$}

In this section we will prove how to reconstruct $\gamma$ from $S(k)$. More precisely we will work with the dual $\tilde{S}(k)=S(-\bar{k})^{T}$, solve the equation (8) for $\tilde{m}$ and finally find $\gamma$ by the formula (11).

Since the equation (8) is mixing terms, we will start by writing pseudoanalytic equation for a suitable linear combination of the elements in $m$ :

Lemma 5.1. Let $z \in \mathbb{C}$ be fixed and let

$$
\tilde{m}_{+}(z, k)=\tilde{m}_{11}(z, k)+\overline{\tilde{m}_{12}(z, \bar{k})}
$$

Then for $k \in \mathbb{C}$ we have the equation

$$
\frac{\partial}{\partial \bar{k}} \tilde{m}_{+}(z, k)=e(z,-k) S_{21}(k) \overline{\tilde{m}_{+}(z, k)}
$$

Proof. The result follows from (8) and (17).

To solve this equation we will need a result about the behavior of $\tilde{m}_{+}$for large $k$ : 
Theorem 5.2 (《Bण97]). Suppose $Q \in L^{p}\left(\mathbb{R}^{2}\right), p>2$ is compactly supported. Then for all $q>2 p /(p-2)$

$$
\sup _{z \in \mathbb{C}}\|m(z, \cdot)-I\|_{L^{q}\left(\mathbb{R}^{2}\right)} \leq C
$$

where $C$ depends on $p, q$ and $Q$.

Furthermore, we will need a certain decay of $S$. In BU97 the fact that $S \in L^{2}\left(\mathbb{R}^{2}\right)$ was sufficient to prove uniqueness of solutions to (23) subject to the asymptotic given in Theorem 5.2. We will however, need a slightly better decay for $S$ in order to write the integral equations corresponding to (23). The following lemma from BBR01 gives the result:

Lemma 5.3. Let $Q \in C^{\epsilon}\left(\mathbb{R}^{2}\right)$ be compactly supported. Then $S \in L^{r}\left(\mathbb{R}^{2}\right)$ for any $r>r_{0}=4 /(2+\epsilon)$.

To simplify notations we introduce the operator $(\partial / \partial \bar{k})^{-1}=\partial_{\bar{k}}^{-1}$ defined by

$$
\partial_{\bar{k}}^{-1} f(k)=\frac{1}{\pi} \int_{\mathbb{C}} \frac{f\left(k^{\prime}\right)}{k-k^{\prime}} d k^{\prime}
$$

We can now write the integral equations for $\tilde{m}_{+}$and prove unique solvability of these:

Lemma 5.4. Let $Q \in C^{\epsilon}\left(\mathbb{R}^{2}\right)$ be compactly supported, and let $z \in \mathbb{C}$ be fixed. Then for $q>4 / \epsilon$ the equations

$$
\left(I-\partial_{\bar{k}}^{-1}\left(e(z,-k) S_{21} \cdot\right)\right)\left(\tilde{m}_{+}-I\right)=\partial_{\bar{k}}^{-1}\left(e(z,-k) S_{21}\right),
$$

have the unique solution $\tilde{m}_{+}(z, \cdot)-I$ in $L^{q}\left(\mathbb{R}^{2}\right)$. Furthermore, $\tilde{m}_{+}(z, \cdot)-I \in C^{\alpha}\left(\mathbb{R}^{2}\right)$, for $\alpha<(1+\epsilon) / 2$.

Proof. Since $S_{21} \in L^{2}\left(\mathbb{R}^{2}\right)$ and $|e(z,-k)|=1$ we know (see Nac93, Lemma 4.2]) that $\partial_{\bar{k}}^{-1}\left(e(z,-k) S_{21} \cdot\right)$ is a compact operator in $L^{s}\left(\mathbb{R}^{2}\right)$ for $2<s<\infty$. Furthermore, since $S_{21} \in L^{r}\left(\mathbb{R}^{2}\right)$ for some $4 /(2+\epsilon)<r<2$ (Lemma 5.3), it follows by the HardyLittlewood-Sobolev inequality $($ Ste70 $)$ that $\partial_{\bar{k}}^{-1}\left(e(z,-k) S_{21}\right) \in L^{q}\left(\mathbb{R}^{2}\right)$ for $q>\epsilon / 2$. Hence the integral equation (24) in $L^{q}\left(\mathbb{R}^{2}\right)$ follows from (23) and Lemma 5.2 .

The unique solvability of (24) in $L^{q}\left(\mathbb{R}^{2}\right)$ follows from Fredholm alternative if $\left(I+\partial_{\bar{k}}^{-1}\left(e(z,-k) S_{21} \cdot\right)\right)$ has trivial kernel in $L^{q}\left(\mathbb{R}^{2}\right)$, but this is known from Liouville's theorem for pseudoanalytic functions since $e(z,-k) S_{21} \in L^{r}\left(\mathbb{R}^{2}\right) \cap L^{r^{\prime}}\left(\mathbb{R}^{2}\right)$ (see BU97, Sun94a, Nac96, Vek62).

To prove the Hölder continuity of $\tilde{m}_{+}(z, \cdot)-I$ we use the fact, that convolution by $1 / z$ maps $L^{p}\left(\mathbb{R}^{2}\right)$ into $C^{\alpha}\left(\mathbb{R}^{2}\right)$ for $1<p<\infty, \alpha=1-1 / p_{0}$ and $\max (2, p)<p_{0}<\infty$, see SU93.

By the previous theorem we find for fixed $z \in \mathbb{C}$

$$
\left(\tilde{m}_{+}(z, k)-I\right)=\left(I-\partial_{\bar{k}}^{-1}\left(e(z,-k) S_{21} \cdot\right)\right)^{-1}\left(\partial_{\bar{k}}^{-1}\left(e(z,-k) S_{21}\right)\right),
$$

in $L^{q}\left(\mathbb{R}^{2}\right) \cap C^{\alpha}\left(\mathbb{R}^{2}\right)$ for $\alpha=1-(1 / r+1 / q)$. To reconstruct $\gamma$ we use the formula

$$
\gamma(z)=\tilde{m}_{11}(z, 0)+\overline{\tilde{m}_{21}(z, 0)}
$$


from [BBR01, Proposition 4.2]. Since $\gamma$ is real, we rewrite the formula using (17) and (22)

$$
\begin{aligned}
\gamma(z) & =\tilde{m}_{11}(z, 0)+\overline{\tilde{m}_{21}(z, 0)} \\
& =\operatorname{Re} \tilde{m}_{11}(z, 0)+\operatorname{Re} \overline{\tilde{m}_{21}(z, 0)} \\
& =\operatorname{Re} \tilde{m}_{11}(z, 0)+\operatorname{Re} \tilde{m}_{12}(z, 0) \\
& =\operatorname{Re} \tilde{m}_{+}(z, 0) .
\end{aligned}
$$

This ends the reconstruction.

\section{APPENDIX}

We will prove, that the Dirichlet to Neumann map for an extended domain $\Omega^{\prime}$ can be calculated from the Dirichlet to Neumann map for the domain $\Omega$ given the value of the conductivity in $\Omega^{\prime} \backslash \bar{\Omega}$ :

Lemma A.1. Let $\gamma \in L^{\infty}(\Omega)$ and let $\Lambda_{\gamma}$ be given. Let $\Omega^{\prime} \subset \mathbb{R}^{2}$ be such that $\bar{\Omega} \subset \Omega^{\prime}$. Take any function $\gamma^{\prime} \in L^{\infty}\left(\Omega^{\prime}\right)$ with $\left.\gamma^{\prime}\right|_{\Omega}=\gamma$.

Then $\Lambda_{\gamma^{\prime}}$ can be recovered from $\Lambda_{\gamma}$ and $\left.\gamma^{\prime}\right|_{\Omega^{\prime} \backslash \bar{\Omega}}$

Proof. From the definition of the Dirichlet to Neumann map we have for any $f, g \in$ $H^{1 / 2}\left(\partial \Omega^{\prime}\right)$, that

$$
\begin{aligned}
\left\langle\Lambda_{\gamma^{\prime}} f, g\right\rangle & =\int_{\Omega^{\prime}} \gamma^{\prime} \nabla u \cdot \nabla v \\
& =\int_{\Omega^{\prime} \backslash \bar{\Omega}} \gamma^{\prime} \nabla u \cdot \nabla v+\left\langle\left.\Lambda_{\gamma} u\right|_{\partial \Omega},\left.v\right|_{\partial \Omega}\right\rangle,
\end{aligned}
$$

where $u \in H^{1}\left(\Omega^{\prime}\right)$ denotes the unique solution to

$$
\begin{aligned}
\nabla \cdot \gamma^{\prime} \nabla u & =0, \text { for } x \in \Omega^{\prime}, \\
u & =f, \text { for } x \in \partial \Omega^{\prime},
\end{aligned}
$$

and $v \in H^{1}\left(\Omega^{\prime}\right)$ is any function with $\left.v\right|_{\partial \Omega^{\prime}}=g$. Hence we see, that $\Lambda_{\gamma^{\prime}}$ can be calculated from $\left.\gamma\right|_{\Omega^{\prime} \backslash \bar{\Omega}}$ and $\Lambda_{\gamma}$ without explicit knowledge of $\gamma$ in $\Omega$, provided the solution $u$ to $(25)$ can be found in $\Omega^{\prime} \backslash \Omega$.

We claim, that $u$ in $\Omega^{\prime} \backslash \Omega$ can be found as the unique solution to

$$
\begin{aligned}
\nabla \cdot \gamma^{\prime} \nabla u & =0, \text { for } x \in \Omega^{\prime} \backslash \bar{\Omega}, \\
u & =f, \text { for } x \in \partial \Omega^{\prime}, \\
\left.\gamma^{\prime} \frac{\partial u}{\partial \nu}\right|_{\partial \Omega} & =\Lambda_{\gamma}\left(\left.u\right|_{\partial \Omega}\right), \text { for } x \in \partial \Omega^{\prime} .
\end{aligned}
$$

That $\left.u\right|_{\Omega^{\prime} \backslash \bar{\Omega}}$ solves (26) follows, since for any $v \in H_{0}^{1}\left(\Omega^{\prime}\right)$

$$
\begin{aligned}
\int_{\Omega^{\prime} \backslash \bar{\Omega}} \gamma^{\prime} \nabla u \cdot \nabla v+\left\langle\left.\Lambda_{\gamma} u\right|_{\partial \Omega},\left.v\right|_{\partial \Omega}\right\rangle & =\int_{\Omega^{\prime}} \gamma^{\prime} \nabla u \cdot \nabla v \\
& =0 .
\end{aligned}
$$


For the uniqueness we assume, that $u_{0} \in H^{1}\left(\Omega^{\prime} \backslash \bar{\Omega}\right)$ solves $(26)$ with $f=0$. Now extend $u_{0}$ into $\Omega$ as the solution to

$$
\begin{aligned}
\nabla \cdot \gamma \nabla u & =0, \text { for } x \in \Omega, \\
u & =u_{0}, \text { for } x \in \partial \Omega .
\end{aligned}
$$

But since $u_{0}$ is in $H^{1}\left(\Omega^{\prime}\right)$ and solves (25) with $f=0$, we conclude, that $u_{0}=0$ in $\Omega^{\prime}$.

\section{REFERENCES}

[Ale88] G. Alessandrini, Stable determination of conductivity by boundary measurements, Appl. Anal. 27 (1988), no. 1-3, 153-172.

[BBR01] J. A. Barceló, T. Barceló, and A. Ruiz, Stability of the inverse conductivity problem in the plane for less regular conductivities, J. Differential Equations 173 (2001), no. 2, 231-270.

[BC85] R. Beals and R. R. Coifman, Multidimensional inverse scatterings and nonlinear partial differential equations, Pseudodifferential operators and applications (Notre Dame, Ind., 1984), Amer. Math. Soc., Providence, RI, 1985, pp. 45-70.

[BC88] R. Beals and R. R. Coifman, The spectral problem for the Davey-Stewartson and Ishimori hierarchies, Nonlinear evolution equations: Integrability and spectral methods, Manchester University Press, Manchester, 1988, pp. 15-23.

[BC89] R. Beals and R. R. Coifman, Linear spectral problems, nonlinear equations and the $\bar{\partial}$ method, Inverse Problems 5 (1989), no. 2, 87-130.

[Bro96] R. M. Brown, Global uniqueness in the impedance-imaging problem for less regular conductivities, SIAM J. Math. Anal. 27 (1996), no. 4, 1049-1056.

[BU96] R. M. Brown and G. Uhlmann, Uniqueness in the inverse conductivity problem with less regular conductivities in two dimensions, Preprint, 1996.

[BU97] R. M. Brown and G. Uhlmann, Uniqueness in the inverse conductivity problem for nonsmooth conductivities in two dimensions, Comm. Partial Differential Equations 22 (1997), no. 5-6, 1009-1027.

[Cal80] A. Calderón, On an inverse boundary value problem, Seminar on Numerical Analysis and its Applications to Continuum Physics (Rio de Janeiro, 1980), Soc. Brasil. Mat., Rio de Janeiro, 1980, pp. 65-73.

[CIN99] M. Cheney, D. Isaacson, and J. Newell, Electrical impedance tomography, SIAM Rev. 41 (1999), no. 1, 85-101 (electronic).

[KV84] R. Kohn and M. Vogelius, Determining conductivity by boundary measurements, Comm. Pure Appl. Math. 37 (1984), no. 3, 289-298.

[KV85] R. Kohn and M. Vogelius, Determining conductivity by boundary measurements. II. Interior results, Comm. Pure Appl. Math. 38 (1985), no. 5, 643-667.

[Liu97] L. Liu, Stability estimates for the two-dimensional inverse conductivity problem, Ph.D. thesis, Department of Mathematics, University of Rochester, New York, 1997.

[Mus53] N. I. Muskhelishvili, Singular integral equations. Boundary problems of function theory and their application to mathematical physics, P. Noordhoff N. V., Groningen, 1953, Translation by J. R. M. Radok.

[Nac88] A. I. Nachman, Reconstructions from boundary measurements, Ann. of Math. (2) 128 (1988), no. 3, 531-576.

[Nac93] A. I. Nachman, Global uniqueness for a two-dimensional inverse boundary value problem, Preprint, 1993.

[Nac96] A. I. Nachman, Global uniqueness for a two-dimensional inverse boundary value problem, Ann. of Math. (2) 143 (1996), no. 1, 71-96.

[NSU88] A. I. Nachman, J. Sylvester, and G. Uhlmann, An n-dimensional Borg-Levinson theorem, Comm. Math. Phys. 115 (1988), no. 4, 595-605. 
[PPU] L. Päivärinta, A. Panchenko, and G. Uhlmann, Complex geometrical optics solutions for Lipschitz conductivities, to appear Rev. Mat. Iberoamericana.

[SMI00] S. Siltanen, J. Mueller, and D. Isaacson, An implementation of the reconstruction algorithm of $A$. Nachman for the $2 D$ inverse conductivity problem, Inverse Problems 16 (2000), no. 3, 681-699.

[Ste70] E. M. Stein, Singular integrals and differentiability properties of functions, Princeton University Press, Princeton, N.J., 1970.

[SU86] J. Sylvester and G. Uhlmann, A uniqueness theorem for an inverse boundary value problem in electrical prospection, Comm. Pure Appl. Math. 39 (1986), no. 1, 91-112.

[SU87] J. Sylvester and G. Uhlmann, A global uniqueness theorem for an inverse boundary value problem, Ann. of Math. (2) 125 (1987), no. 1, 153-169.

[SU88] J. Sylvester and G. Uhlmann, Inverse boundary value problems at the boundarycontinuous dependence, Comm. Pure Appl. Math. 41 (1988), no. 2, 197-219.

[SU93] Z. Sun and G. Uhlmann, Recovery of singularities for formally determined inverse problems, Comm. Math. Phys. 153 (1993), no. 3, 431-445.

[Sun94a] L. Sung, An inverse scattering transform for the Davey-Stewartson II equations. I, J. Math. Anal. Appl. 183 (1994), no. 1, 121-154.

[Sun94b] L. Sung, An inverse scattering transform for the Davey-Stewartson II equations. II, J. Math. Anal. Appl. 183 (1994), no. 2, 289-325.

[Sun94c] L. Sung, An inverse scattering transform for the Davey-Stewartson II equations. III, J. Math. Anal. Appl. 183 (1994), no. 3, 477-494.

[Uhl99] G. Uhlmann, Developments in inverse problems since Calderón's foundational paper, Harmonic analysis and partial differential equations (Chicago, IL, 1996), Univ. Chicago Press, Chicago, IL, 1999, pp. 295-345.

[Vek62] I. N. Vekua, Generalized analytic functions, Pergamon Press, London, 1962.

Kim Knudsen, Department of Mathematical Sciences, Aalborg University, Fredrik Bajers Vej 7G, DK-9220 Aalborg Ø, Denmark.

E-mail address: kim@math.auc.dk

Alexandru Tamasan, Department of Mathematics, University of Washington, Box 354350, Seattle, Wa 98195-4350

E-mail address: tamasan@math.washington.edu 